\title{
Note on a Conjecture of Sierksma*
}

\author{
Aleksandar Vučić ${ }^{1}$ and Rade T. Živaljević \\ ${ }^{1}$ Faculty of Mathematics, Studentski trg 16, \\ 11000 Beograd, Yugoslavia \\ ${ }^{2}$ Mathematics Institute, Knez Mihailova 35/1, p.f. 367. \\ 11001 Beograd, Yugoslavia \\ ezivalje@ yubgef 51 .bitnet
}

\begin{abstract}
Let $S(q, d)$ be the maximal number $v$ such that, for every general position linear map $h: \Delta^{(q-1)(d+1)} \rightarrow R^{d}$, there exist at least $v$ different collections $\left\{\Delta^{t_{1}}, \ldots, \Delta^{t_{q}}\right\}$ of disjoint faces of $\Delta_{(q-1)(d+1)}$ with the property that $f\left(\Delta^{t}\right) \cap \cdots \cap f\left(\Delta^{t_{q}}\right) \neq \varnothing$. Sierksma's conjecture is that $S(q, d)=((q-1) !)^{d}$. The following lower bound (Theorem 1) is proved assuming that $q$ is a prime number:
\end{abstract}

$$
S(q, d) \geq \frac{1}{(q-1) !}\left(\frac{q}{2}\right)^{((q-1)(d+1)) / 2} .
$$

Using the same technique we obtain (Theorem 2) a lower bound for the number of different splittings of a "generic" necklace.

\section{Introduction}

The well-known Tverberg theorem [15] has attracted much interest among combinatorial geometers in the last 10 years. This theorem, which was itself inspired by Radon's theorem, a close relative of Helly's convexity theorem, has inspired much research and nowadays is surrounded by a growing family of related results. In its original form the theorem claims that every $A \subset R^{d}$ of size $(q-1)(d+1)+1$ permits a partition $A=A_{1} \cup \cdots \cup A_{q}$ such that $\bigcap_{i=1}^{q} \operatorname{conv}\left(A_{i}\right) \neq \varnothing$. The revival of this theorem began when Bárány et al. [4] first

\footnotetext{
* Work on this paper was supported by the Mathematical Institute, Belgrade, through the Serbian Science Foundation under Grant No. 0401D.
} 
observed that it can be formulated as a statement about a linear map $h: \Delta^{(p-1)(d+1)} \rightarrow R^{d}$ and then proved by topological methods that, for any prime number $p$ and a continuous map $f$, there exist disjoint faces

$$
\Delta^{t_{1}}, \ldots, \Delta^{t_{p}} \subset \Delta^{(p-1)(d+1)}
$$

such that $\bigcap_{i=1}^{p} f\left(\Delta^{t_{1}}\right) \neq \varnothing$. Naturally, it is hoped that the condition on $p$ above is superficial but so far, in spite of several attempts, it seems to be unknown whether this statement is true for every continuous map $f: \Delta^{(q-1)(d+1)} \rightarrow R^{d}$ and every $q$ and $d$. Ozaydin has announced that the theorem is true if $q$ is a power of a prime number [10]. A new and interesting approach was proposed in a preprint by Sarkaria [12]. He very skillfully used the technique of deleted joins combined with the obstruction theory, with the main goal of proving a general form of Tverberg's theorem for all $q$, including a result that there exist at least $((q-1) !)^{d}$ different partitions with the desired property. Unfortunately, Sarkaria's original and beautiful argument had a serious gap so the problem, known as Sierksma's Dutch cheese problem, see [11], of finding how many different partitions there are in Tverberg's theorem is still open.

Let us denote by $S(q, d)$ the maximal number $v$ such that, for every general position linear map $h: \Delta^{(q-1)(d+1)} \rightarrow R^{d}$, there exist at least $v$ different collections (partitions) $\left\{\Delta^{t_{1}}, \ldots, \Delta^{t_{q}}\right\}$ of disjoint faces of $\Delta^{(q-1)(d+1)}$ with the property $h\left(\Delta^{i}\right) \cap \cdots \cap h\left(\Delta^{t_{q}}\right) \neq \varnothing$. Then Sierksma's conjecture is that $S(q, d)=((q-1) !)^{d}$.

The main objective of our note is to provide a lower bound for $S(q, d)$ under the condition that $q$ is an odd prime number. In fact, since we use a topological argument, our bound

$$
S(q, d) \geq \frac{1}{(q-1) !}\left(\frac{q}{2}\right)^{((q-1)(d+1)) / 2},
$$

established in Theorem 1, holds not only for a linear but also for a general position continuous map $f: \Delta^{(q-1)(d+1)} \rightarrow R^{d}$. Also, using the same technique we obtain a lower bound for the number of different splittings of a "generic" open necklace.

\section{Configuration Spaces and the Main Theorem}

The main result of this paper is the following theorem. Recall that $S(q, d)$ is the maximal number $v$ such that, for every general position linear map $h: \Delta^{(q-1)(d+1)} \rightarrow R^{d}$, there exist at least $v$ different collections (partitions) $\left\{\Delta^{t_{1}}, \ldots, \Delta^{t_{q}}\right\}$ of disjoint faces of $\Delta^{(q-1)(d+1)}$ with the property $f\left(\Delta^{t_{1}}\right) \cap \cdots \cap f\left(\Delta^{t_{q}}\right) \neq$ $\varnothing$. Here, $h$ is a general position linear map if the image of the set of extreme points of $\Delta^{(q-1)(d+1)}$ is a set of points in general position in the usual sense.

Theorem 1. Let $q$ be an odd prime number. Then

$$
S(q, d) \geq \frac{1}{(q-1) !} \cdot\left(\frac{q}{2}\right)^{\Lambda / 2}, \quad \Lambda=(q-1)(d+1) .
$$


Moreover, if $f: \Delta^{\wedge} \rightarrow R^{d}$ is an arbitrary continuous map which is assumed to be in general position in the sense of Definition 1 , then there exist at least

$$
\frac{1}{(q-1) !} \cdot\left(\frac{q}{2}\right)^{\Lambda / 2}
$$

different collections ( partitions) $\left\{\Delta^{t_{1}}, \ldots, \Delta^{t_{q}}\right\}$ of disjoint faces of $\Delta^{\wedge}$ with the property

$$
f\left(\Delta^{t_{1}}\right) \cap \cdots \cap f\left(\Delta^{t_{q}}\right) \neq \varnothing
$$

For comparison, let us note that, for $q=3$, the estimate above gives $\frac{1}{2}\left(\frac{3}{2}\right)^{d+1}$ desired partitions whereas Sierksma's conjecture is that there should be at least $2^{d}$ of them.

The proof of Theorem 1, given in Section 3, is based on a sequence of lemmas about configuration spaces which naturally arise in this context. Since these spaces play a central role in any known result related to Tverberg's problem, we briefly review relevant definitions and facts.

Tverberg's problem or any of its variations can be rephrased as the search for $q$-tuples of points $x_{1}, \ldots, x_{q} \in \Delta^{\Lambda}, \Lambda=(q-1)(d+1)$, which belong to disjoint faces of $\Delta^{\wedge}$, such that $f\left(x_{1}\right)=\cdots=f\left(x_{q}\right)$. A $q$-tuple of points having the property that the smallest faces of $\Delta^{\Lambda}$, where they belong, are pairwise disjoint, are called separated. They are called good q-tuples for the function $f$, or simply good q-tuples, if they are separated and $f\left(x_{1}\right)=\cdots=f\left(x_{q}\right)$. A natural first step in approaching the problem of finding good $q$-tuples is to collect together all separated $q$-tuples in a configuration space and then analyze its relevant topological properties. There are two natural possibilities tied with the so-called deleted product and deleted join construction. Let $K$ be any simplicial complex. Then $K_{\Delta}^{q}$ and $K_{\Delta}^{(q)}$, the $q$ th deleted product and the $q$ th deleted join of $K$, respectively, are seen as subspaces of the product $K^{q}$ and the join $K^{(q)}$ defined by

$$
\begin{aligned}
K_{\Delta}^{q} & =\bigcup\left\{\sigma_{1} \times \cdots \times \sigma_{q} \mid \sigma_{i} \in K \&(\forall i \neq j) \sigma_{i} \cap \sigma_{j}=\varnothing\right\} \subset K^{q}, \\
K_{\Delta}^{(q)} & =\bigcup\left\{\sigma_{1} * \cdots * \sigma_{q} \mid \sigma_{i} \in K \&(\forall i \neq j) \sigma_{i} \cap \sigma_{j}=\varnothing\right\} \subset K^{(q)} .
\end{aligned}
$$

It is useful to use both of these constructions, although it is worth mentioning that, in spite of the fact that the definition of $K_{\Delta}^{q}$ looks more natural, $K_{\Delta}^{(q)}$ seems to have some other advantages. A first and obvious advantage is that $K_{\Delta}^{(q)}$ is a simplicial complex and $K_{\Delta}^{q}$ is not. Nevertheless, both of these constructions are useful and they have been successfully applied, for example, the first in [4] and the second in [13] and [16].

Let $J_{q, d}=\left(\Delta^{\wedge}\right)_{\Delta}^{(q)}$. An important topological property, that is usually of interest when dealing with configuration spaces, is their conectivity. For example, a key technical fact proved in $[4]$ is that $\left(\Delta^{\Lambda}\right)_{\Delta}^{q}$ is $((q-1) d-1)$-connected. It turns out that, from this point of view, deleted joins are much easier to handle, see, e.g., [13] or [16]. For this purpose we formulate the following easy but fundamental proposition. 
Proposition 1. The deleted join operation commutes with joins, i.e., for two simplicial complexes $K$ and $L$,

$$
(K * L)_{\Delta}^{(q)} \cong K_{\Delta}^{(q)} * L_{\Delta}^{(q)}
$$

Proof. The proof follows immediately from the observation that the (abstract) simplexes in $K * L$ are of the form $\tau \cup \sigma$ for some $\tau \in K$ and $\sigma \in L$ and the simplexes in $K^{(q)}$ are unions $\sigma_{1} \cup \cdots \cup \sigma_{q}$ where $\sigma_{i} \in K_{i}$ and $K_{i}, i=1, \ldots, q$, are disjoint copies of $K$.

Equipped with the proposition above a better picture of deleted joins of complexes which are joins themselves can often be obtained. Specially,

$$
J_{q, d}=\left((p t)^{(\Lambda+1)}\right)_{\Delta}^{(q)} \cong\left((p t)_{\Delta}^{(q)}\right)^{(\Lambda+1)} \cong[q]^{(\Lambda+1)},
$$

where $[q]=\{1,2, \ldots, q\}$ and $p t$ is a one-point space. The last complex is easily seen to be isomorphic to the simplicial complex $\Omega(\Lambda+1, q)$, where

$$
\Omega(m, n)=\{f \subset[m] \times[n] \mid f \neq \varnothing \& f \text { is a function }\} .
$$

Here, functions are seen as sets of ordered pairs, i.e., functions and their graphs are not distinguished. We call a function $f$ maximal if $\operatorname{dom} f=[m]$.

Alternatively, $\Omega(m, n)$ can be described as the simplicial complex defined on the $m \times n$ chessboard, where simplexes are those rook placements with the property that no two rooks are placed in the same column.

It is well known, see [5], [7] or [8], that $\Omega(m, n)$ is $(\operatorname{dim} \Omega(m, n)-1)$-connected. Hence, $J_{q, d}$ is $(\Lambda-1)$-connected. In the special case when $q=2$ we observe that $J_{2, d}$ is actually the hyperoctahedral triangulation of the $(d+1)$-dimensional sphere.

We can embed the sphere $S^{\Lambda} \cong \Omega(\Lambda+1,2)$ in $J_{q, d}$, for any $q \geq 2$, by specifying two maximal functions $f, g \in \Omega(\Lambda+1, q) \cong J_{q, d}$ such that $f(i)<g(i)$ for all $1 \leq i \leq \Lambda+1$; this sphere is denoted by $S(f, g)$. A maximal function $h \in J_{q, d}$ belongs to the triangulation of $S(f, g)$ iff $h(i) \in\{f(i), g(i)\}$ for all $i$. It turns out that this construction provides a convenient basis for the homology $H_{\Lambda}\left(J_{q, d}\right)$ but we omit the details since this fact is not needed in what follows.

For every continuous map $f: \Delta^{\Lambda} \rightarrow R^{d}$, let

$$
F=f^{(q)}: J_{q, d}=\left(\Delta^{\Lambda}\right)_{\Delta}^{(q)} \rightarrow\left(R^{d}\right)^{(q)}
$$

be the natural map induced by passing to joins. Note that $F$ is a $Z_{q}$-equivariant continuous map. Moreover, the elements $x \in J_{q, d}$ with $F(x) \in D$ are in one-to-one correspondence with (ordered) good $q$-tuples, where $D$ is the diagonal in $\left(R^{d}\right)^{(q)}$ defined by

$$
D=\left\{t_{1} x_{1}+\cdots+t_{q} x_{q} \in\left(R^{d}\right)^{(q)} \mid(\forall i, j) t_{i}=1 / q \& x_{i}=x_{j}\right\} .
$$


Therefore, every good $q$-tuple determines $q$ ! elements from the set

$$
\left\{x \in J_{q, d} \mid F(x) \in D\right\} .
$$

Note that $\left(R^{d}\right)^{(q)}$ is a $Z_{q}$-space $\left(Z_{q}\right.$-action permutes the coordinates) and becomes a free $Z_{q}$-space after removing the diagonal

Definition 1. A map $f: \Delta^{\Lambda} \rightarrow R^{d}$ is in general position if $f^{(q)}\left(J_{q, d}^{(-1)}\right) \cap D=\varnothing$, where $J_{q, d}^{(-1)}$ is the codimension one skeleton of the complex $J_{q, d}$. In other words, $f$ is in general position if no good $q$-tuple is contained in the boundary of a topdimensional simplex of $J_{q, d}$.

It can be easily checked that if $f$ is a general position linear map, then $f$ is in general position in the sense of Definition 1.

The proof of Theorem 1, given in Section 3, utilizes a result (Proposition 3) about degrees of $Z_{q}$-equivariant maps between free $Z_{q}$-spheres. This result will be applied to $Z_{q}$-spheres $\Sigma_{\mathscr{P}}$ which naturally arise in connection with the configuration space $J_{q, d}$. All these spheres are homologically trivial in $J_{q, d}$, i.e., they induce trivial classes in $H_{*}\left(J_{q, \mathrm{~d}}\right)$ and they come naturally with cones

$$
\Theta_{\mathscr{P}, v}=\{v\} * \Sigma_{\mathscr{P}} \subset J_{q, d} .
$$

The following proposition gives a description and an estimate of the number of different cones over free, $Z_{q}$-invariant, $(\Lambda-1)$-dimensional spheres in $J_{q, d}$.

Proposition 2. Let $q$ be an odd prime number. Then there exists at least

$$
\varphi=(\Lambda+1) \cdot N \cdot q \cdot\left(\begin{array}{l}
q \\
2
\end{array}\right)^{\Lambda / 2}
$$

different top-dimensional subcomplexes of $J_{q, d}$ which are cones over free $Z_{q}$-equivariant spheres. Here and later $N$ denotes the number of partitions of $[\Lambda]$ in pairs.

Proof. There is a one-dimensional invariant sphere in $\Omega(2, q)$ for every unordered pair $\{u, v\}$ of numbers $1 \leq u<v \leq q$; we call such a pair admissible. It is described as the unique equivariant circle in $\Omega(2, q)$ which contains 1 -simplices $\{(1,1),(2, u)\}$ and $\{(1,1),(2, v)\}$. All these circles, denoted by $S_{u, v}$, are different. For every pair of numbers $1 \leq i<j \leq \Lambda+1$ and an admissible pair $\{u, v\}$, let $S_{u, v}^{i, j}$ be the circle described above with 1,2 replaced by $i$ and $j$.

We choose an integer $\gamma \in[\Lambda+1]$, a partition $\pi$ of $[\Lambda+1] \backslash\{\gamma\}$ into pairs $\left\{i_{1}, j_{1}\right\}$, $\left\{i_{2}, j_{2}\right\}, \ldots,\left\{i_{s}, j_{s}\right\}, 2 s=\Lambda$, and admissible pairs $\left\{u_{k}, v_{k}\right\}=\varphi(k), 1 \leq k \leq s$. Let

Let $\mathscr{P}=(\gamma, \pi, \varphi)$ denote this choice and let $\mathscr{C}$ be the set of all possible choices.

$$
\Sigma_{\mathscr{P}}=S_{u_{1}, v_{1}}^{i_{1}, j_{1}} * S_{u_{2}, v_{2}}^{i_{2}, j_{2}} * \cdots * S_{u_{4,}, v_{1}}^{i_{1}, j}
$$


be the $[\Lambda-1]$-dimensional sphere associated with $\mathscr{P}$. Then $\Sigma_{\mathscr{P}}$ is a $Z_{q}$-invariant subcomplex of $J_{q, \alpha}$ and if $\mathscr{P}, \mathscr{P} \in \mathscr{C}$ are different, then the spheres $\Sigma_{\mathscr{P}}$ and $\Sigma_{\mathscr{P} \text {, are }}$ also different. Choose a point $v \in\{\gamma\} \times[q]$. Then $\Theta_{\mathscr{P}, v}=\{v\} * \Sigma_{\mathscr{P}}$ is a cone over $\Sigma_{\mathscr{P}}$ with vertex $v$.

A simple calculation shows that the number of these cones is

$$
\beta=(\Lambda+1) \cdot N \cdot q \cdot\left(\begin{array}{l}
q \\
2
\end{array}\right)^{\Lambda / 2}
$$

Remark 1. First note that the action of $Z_{q}$ on the circle $S_{u, v}^{i, j}$ is equivalent to the action on $S^{1} \subset R^{2}$ induced by the rotation through an angle $2 \pi \mathrm{m} / q$, where $m(u-v) \equiv 1(\bmod q)$. In other words, for some orthogonal action of $Z_{q}$ on $S^{1} \subset R^{2}$, there exists a $Z_{q}$-equivariant homeomorphism $\alpha: S_{u, v}^{i, j} \rightarrow S^{1}$. Since the join of two orthogonal actions is also orthogonal, we conclude that the action of $Z_{q}$ on $\Sigma_{\mathscr{P}}$ is also equivalent to an orthogonal action.

Remark 2. Proposition 2 remains true even if we omit the assumption that $q$ is an odd prime number. In that case, assuming only that $\Lambda$ is an even number, we obtain

$$
\beta=(\Lambda+1) \cdot N \cdot q \cdot\left(\frac{q \varphi(q)}{2}\right)^{\Lambda / 2}
$$

where $\varphi(n)$ is the number of integers smaller than $n$ and relatively prime to $n$.

We assumed in Proposition 2 that $q$ is a prime number since it simplified the presentation and we need this assumption later in order to guarantee that certain actions on spheres are free.

\section{Equivariant Maps Between Spheres}

Let $S^{n}$ be a sphere equipped with a free action of the group $Z_{m}$, where $m$ is not necessarily a prime number. Then, for every $Z_{m}$-equivariant map $f: S^{n} \rightarrow S^{n}$, $\operatorname{deg}(f)=1(\bmod m)$. This simple and elegant result, proved by Krasnoselsky and Zabrejko in [9], was a key ingredient in the proof of the continuous Tverberg theorem [4]. We need a more general result which applies to $Z_{m}$-equivariant maps $f: S^{n} \rightarrow S^{n}$ between free $Z_{m}$-spheres which are not necessarily the same as $Z_{m^{-}}$ spaces. Before we formulate this result, let us recall some necessary definitions and facts about group actions and equivariant maps.

Let $Z_{m}$ be the cyclic group, say $Z_{m}=\left\{\zeta^{k} \mid k \in Z, \zeta=\exp (2 \pi i / m)\right\}$. Given positive integers $r_{1}, \ldots, r_{n}$ prime to $m$, the complex representation $V(\vec{r})=V\left(r_{1}, \ldots, r_{n}\right)$ is defined by

$$
Z_{m} \times C^{n} \rightarrow C^{n}, \quad\left(\zeta,\left(z_{1}, \ldots, z_{n}\right)\right) \mapsto\left(\zeta^{r_{1}} z_{1}, \ldots, \zeta^{r_{n}} z_{n}\right)
$$


Let $S(V)$ be the unit sphere in the Euclidean vector space $V$. So,

$$
S V(\vec{r})=S V\left(r_{1}, \ldots, r_{n}\right)
$$

is the unit sphere in $V(\vec{r})$. For a fixed $n$, we choose an orientation on $C^{n}$ which induces an orientation on $S\left(C^{n}\right)$ so the degree $\operatorname{deg}(f)$ is well defined for every map $f: S V(\vec{r}) \rightarrow S V(\vec{s})$. The proof of the following proposition can be found in Proposition II.4.12 of [14].

Proposition 3. For every $Z_{m}$-equivariant map $f: S V\left(r_{1}, \ldots, r_{n}\right) \rightarrow S V\left(s_{1}, \ldots, s_{n}\right)$, the degree $\operatorname{deg}(f)$ of $f$ satisfies the relation

$$
\operatorname{deg}(f) r_{1} \cdots r_{n} \equiv s_{1} \cdots s_{n} \quad(\bmod m) .
$$

Corollary 1. For every equivariant map $f: S V\left(r_{1}, \ldots, r_{n}\right) \rightarrow S V\left(s_{1}, \ldots, s_{n}\right), \operatorname{deg}(f) \neq 0$.

Proposition 4. Let $V$ and $W$ be real Euclidean spaces equipped with an orthogonal action of the group $Z_{m}$ such that the induced actions on $S(V)$ and $S(W)$ are free. Then, for every $Z_{m}$-equivariant map $f: S(V) \rightarrow S(W), \operatorname{deg}(f) \neq 0$.

Proof. $V \oplus V$ and $W \oplus W$ can be identified, as real vector spaces, with the complexifications of $V$ and $W$, respectively. Since the actions of $Z_{m}$ on $V$ and $W$ are orthogonal and $O(n) \subset U(n)$, we observe that the induced actions on $V \oplus V$ and $W \oplus W$ are unitary and free. Hence,

$$
S(V \oplus V) \cong S(V \otimes C)(\vec{r}) \quad \text { and } \quad S(W \oplus W) \cong S(W \otimes C)(\vec{s})
$$

as $Z_{m}$-spaces for some $\vec{r}$ and $\vec{s}$. Also, as $Z_{m}$-spaces $S(V \oplus V) \cong S(V) * S(V)$ and by Corollary 1 applied to the equivariant map $f * f: S(V \oplus V) \rightarrow S(W \oplus W)$, $\operatorname{deg}(f * f) \neq 0$ which implies $\operatorname{deg}(f) \neq 0$.

\section{Proof of Theorem 1}

Proof of Theorem 1. Let $F=f^{(q)}: J_{q, d} \rightarrow\left(R^{d}\right)^{(q)}$ be the map induced by $f: \Delta^{\Lambda} \rightarrow R^{d} . F$ is a $Z_{q}$-equivariant continuous map. Moreover, the elements $x \in J_{q, d}$ with $F(x) \in D$ are in one-to-one correspondence with (ordered) good $q$-tuples, where $D$ is the diagonal in $\left(R^{d}\right)^{(q)}$ defined by

$$
D=\left\{t_{1} x_{1}+\cdots+t_{q} x_{q} \in\left(R^{d}\right)^{(q)} \mid(\forall i, j) t_{i}=1 / q \& x_{i}=x_{j}\right\} .
$$

Therefore, every good $q$-tuple determines $q$ ! elements from the set

$$
\left\{x \in J_{q, d} \mid F(x) \in D\right\} \text {. }
$$

Note that $\left(R^{d}\right)^{(q)}$ is a $Z_{q}$-space $\left(Z_{q}\right.$-action permutes coordinates) and becomes a free $Z_{q}$-space after removing the diagonal. So, all we need, in order to complete 
the proof of Theorem 1 , is the following proposition about $Z_{q}$-equivariant maps $F: J_{q, d} \rightarrow\left(R^{d}\right)^{(q)}$.

Proposition 5. Let $F: J_{q, d} \rightarrow\left(R^{d}\right)^{(q)}$ be a $Z_{q}$-equivariant continuous map such that

$$
F\left(J_{q, d}^{(-1)}\right) \cap D=\varnothing
$$

where $J_{q, d}^{(-1)}$ is the codimension one skeleton of $J_{q, d}$. Then

$$
\operatorname{card}\left\{x \in J_{q, d} \mid F(x) \in D\right\} \geq q \cdot\left(\frac{q}{2}\right)^{\wedge / 2} .
$$

Proof. From (1) we have that every $x \in A:=\left\{x \in J_{q, d} \mid F(x) \in D\right\}$ belongs to the interior of some top-dimensional simplex of $J_{q, d}$. Take a cone $\Theta_{\mathscr{Q}, v}$ over one of the spheres $\Sigma_{\mathscr{P}}$, constructed in the proof of Proposition 2. Then the composition of

$$
\left.F\right|_{\Sigma_{\mathscr{P}}}: \Sigma_{\mathscr{P}} \rightarrow\left(R^{d}\right)^{(q)} \backslash D
$$

with the $Z_{q}$-homotopy equivalence $\left(R^{d}\right)^{(q)} \backslash D \simeq S\left(D^{1}\right)$ has a nonzero degree (Proposition 4 and Remark 1). From here we deduce

$$
F\left(\Theta_{\mathscr{P}, v}\right) \cap D \neq \varnothing .
$$

Hence, every cone $\Theta_{\mathscr{P}, v}$ contains at least one element from $A$. Of course, each element from $A$ appears in many cones. More precisely, every top-dimensional simplex from $J_{q, d}$, consequently every element from $A$, appears in exactly

$$
\psi=(\Lambda+1) \cdot N \cdot(q-1)^{\Lambda / 2}
$$

cones. Indeed, let us take a top-dimensional simplex, i.e., a maximal function

$$
f \in \Omega(\Lambda+1, q) \cong J_{q, d}
$$

There are $\Lambda+1$ choices for the vertex of a cone containing the maximal simplex determined by $f$. The column $\{\gamma\} \times[q]$ containing this vertex is uniquely determined by $f$. Also, we have $N$ different splittings of the set $[\Lambda+1] \backslash\{\gamma\}$ in pairs, and, for each pair, $(q-1)$ choices for an admissible pair (one element from the admissible pair is already determined by $f$ ). Hence,

$$
\operatorname{card}(A) \geq \frac{\varphi}{\psi}=q \cdot\left(\frac{q}{2}\right)^{((q-1)(d+1)) / 2} .
$$

\section{Application to the Splitting Necklace Problem}

It was a beautiful observation of Alon [1], that the so-called "splitting necklace problem" requires for its solution the method of equivariant maps. His solution 
was based on the continuous Tverberg theorem of Bárány et al., so it is not a surprise that the method, which gave us a lower bound for Sierksma's cheese problem, also gives a lower bound for the number of different fair splittings of a generic open necklace. Let us remind ourselves about the necklace problem. A (continuous) model of an open necklace is the unit interval $I=[0,1]$ together with disjoint measurable sets $A_{1}, \ldots, A_{d} \subset I$ representing beads of different colors. The unit interval has to be cut in pieces and the pieces distributed to $q$ different persons. This distribution is called a splitting. The question is to find the least number of cuts needed for a fair splitting which means that each person should receive a $w$ th part of the measure of each of the sets $I, A_{1}, \ldots, A_{d}$.

The answer to this problem, given by Alon [1], is that $\Lambda=(q-1)(d+1)$ cuts are sufficient.

The following question could also be raised:

Question 1. Assuming that the necklace is in general position, which means that there are no fair splittings with fewer than $(q-1)(d+1)$ cuts, what is the lower bound for the number of different fair splittings with $\Lambda=(q-1)(d+1)$ cuts?

The following analysis shows that the deleted join technique is very suitable for describing splittings of a necklace. This leads to a simpler proof of Alon's theorem and to Theorem 2, which gives a lower bound for a number of splittings of a generic necklace.

We start with an observation that the space of all cuts of an open necklace into at most $\Lambda$ small intervals is isomorphic to $\Delta^{\Lambda}$. The vertices of $\Delta^{\Lambda}$ correspond to $\Lambda+1$ small intervals in which $I$ is divided and the coordinates are lengths of the intervals. A splitting is obtained by arranging $\Lambda+1$ small intervals into $q$ groups, which implies that the space of all splittings is isomorphic to $J_{q, d}=\left(\Delta^{(q-1)(d+1)}\right)_{\Delta}^{(q)}$. More precisely, suppose we have a splitting $S$, i.e., a division of $I$ into intervals $I_{1}, \ldots, I_{\Lambda+1}$. For each interval it has to be decided to whom the interval belongs. Every choice is nothing else but a maximal function $f \in \Omega(\Lambda+1, q)$, or in other words, we are given a top-dimensional simplex from $J_{q, d}$. Let $I_{1}^{i}, \ldots, I_{n_{i}}^{i}$ be the intervals given to the $i$ th person. Let $m_{i}=m\left(I_{1}^{i}\right)+\cdots+m\left(I_{n}^{i}\right)$, where $m\left(I_{j}^{i}\right)$ is the length of the interval $I_{j}^{i}$. Then the element from $J_{q, d}$, associated with a given splitting is

$$
x(S)=m_{1}\left(\frac{m\left(I_{1}^{1}\right)}{m_{1}} I_{1}^{1}+\cdots+\frac{m\left(I_{n_{1}}^{1}\right)}{m_{1}} I_{n_{1}}^{1}\right)+\cdots+m_{q}\left(\frac{m\left(I_{1}^{q}\right)}{m_{q}} I_{1}^{q}+\cdots+\frac{m\left(I_{n_{q}}^{q}\right)}{m_{q}} I_{n_{q}}^{q}\right) .
$$

The map $\Psi$ : $\{$ subintervals of $I\} \rightarrow R^{d}$ given by

$$
\Psi(L)=\left(m\left(L \cap A_{1}\right), \ldots, m\left(L \cap A_{d}\right)\right)
$$

computes the vector of values of all beads contained in a given interval. Now we define a map $F: J_{q, d} \rightarrow\left(R^{d}\right)^{(q)}$ by

$$
F(x(S))=m_{1}\left[\Psi\left(I_{1}^{1}\right)+\cdots+\Psi\left(I_{n(1)}^{1}\right)\right]+\cdots+m_{q}\left[\Psi\left(I_{1}^{q}\right)+\cdots+\Psi\left(I_{n(q)}^{q}\right)\right] .
$$


This map obviously measures how far from being fair is a given splitting. $F$ is a $Z_{q}$-equivariant continuous map and a splitting $S$ is fair iff $F(x(S)) \in D$. Finally, note that a necklace is in general position iff $F$ satisfies condition (2).

So, everything is set up for an application of Proposition 5 which implies the following result.

Theorem 2. Let $q$ be an odd prime. Then if a necklace is generic in the sense that it does not admit a fair splitting consisting of less than $\Lambda=(q-1)(d+1)$ cuts, then there are at least

$$
q \cdot\left(\frac{q}{2}\right)^{\Delta / 2}
$$

fair splittings with $\Lambda$ cuts.

Remark 3. In case $q$ is not a prime number a nontrivial lower bound can still be found for the number of fair splittings of a generic necklace. For this purpose the observation already used in Proposition 3.3 of [2], can be used to obtain a fair splitting in the case $q=u \cdot v$ if it is known how to split a necklace fairly in cases $q=u$ and $q=v$.

Remark 4. Theorem 2 remains true if the sets $A_{1}, \ldots, A_{d}$ and the Lebesgue measure on $[0,1]$ are replaced by $d+1$ nonatomic, $\sigma$-additive Borel measures on the unit interval.

Let us conclude this section with the following problem.

Question 2. Design an algorithm for finding a top-dimensional simplex containing a good $q$-tuple.

Alon [2] has already raised the question of finding an algorithm for designing a fair splitting of a necklace. A positive answer to Question 3 would provide a first step in this direction. After that, methods of linear programming would probably be applied to obtain a fair splitting.

The proof of Lemma 1 shows that every cone $\Theta_{\mathscr{P}, v}$ contains a good $q$-tuple. Hence, the algorithm requested in Question 3 may take into account only those simplexes which belong to a fixed cone $\Theta_{\mathscr{P}, v}$.

\section{Acknowledgments}

We would like to thank Siniša Vrecica, Imre Bárány, and the referee for useful suggestions and remarks. 


\section{References}

1. N. Alon, Splitting necklaces, Adv. in Math. 63 (1987), 247-253.

2. N. Alon, Some recent combinatorial applications of Borsuk-type theorem, in Algebraic, Extremal and Metric Combinatorics, M. Deza, P. Frankl, and D. G. Rosenberg (eds.), Cambridge University Press, Cambridge, pp. 1-12.

3. I. Bárány, Geometric and combinatorial applications of Borsuk's theorem, A survey, preprint.

4. I. Bárány, S. B. Shlosman, and A. Szücs, On a topological generalization of a theorem of Tverberg, J. London Math. Soc. (2) 23 (1981), 158-164.

5. A. Björner, Shellable and Cohen-Macaulay partially ordered sets, Trans. Amer. Math. Soc. 260 (1980), 159-183.

6. A. Björner, Topological methods, in Handbook of Combinatorics, R. Graham, M. Grötschel, and L. Lovász, (eds.), in press.

7. A. Björner and M. Wachs, On lexicographically shellable posets, Trans. Amer. Math. Soc. 277 (1983), 323-341.

8. L. Budach, B. Graw, C. Meinel, and S. Waack, Algebraic and Topological Properties of Finite Partially Ordered Sets, Teubner, Leipzig, 1988.

9. M. A. Krasnoselsky and P. P. Zabrejko, Geometritshskie zatatshi nelinejnogo analiza, Nauka, Moscow, 1975.

10. M. Ozaydin, to appear.

11. J. R. Reay, Open problems around Radon's theorem, in Convexity and Related Problems in Combinatorial Geometry, D. C. Kay and M. Breen (eds.), Marcel Dekker, New York, 1982, pp. 151-171.

12. K. S. Sarkaria, Sierksma's "Dutch cheese problem", preprint.

13. K. S. Sarkaria, Kuratowski complexes, Topology, to appear.

14. T. tom Dieck, Transformation Groups, Studies in mathematics, Vol. 8, De Gruyter.

15. H. Tverberg, A generalization of Radon's theorem, J. London Math. Soc. 41, 123-128.

16. R. T. Živaljevic and S. T. Vrecica, The colored Tverberg's problem and complexes of injective functions, J. Combin. Theory Ser. A, to appear.

Received October 3, 1991, and in revised form December 13, 1991, and August 31, 1992. 